\title{
Convictions et faits
}

\author{
Paul Robel, Alain Jardin
}

L 'article de F. Labrie est un article d'opinion.

Il condense un travail publié par lui dans un numéro spécial du Joumal of Urology intitulé : "serum prostate specific antigen as prescreening test for prostatic cancer ". Cette étude avait été considérée par tous comme contributive puisque le dépistage portait sur mille sujets choisis au hasard parmi une population d'hommes de 45 à 80 ans. La répartition par tranches d'âge de ces mille hommes n'était d'ailleurs pas donnée. A partir de cette étude qui a permis de mettre en évidence 57 cancers de prostate, F. Labrie propose une stratégie qu'il dit efficace et peu coûteuse pour la détection du cancer de la prostate à un stade précoce alors que la guérison est possible. Cette stratégie repose sur la seule pratique systématique dans toute la population du dosage de PSA.

Comme base de départ de son raisonnement, F. Labrie affirme qu'il existe une fenêtre de quelques années permettant de mettre en évidence le cancer de la prostate avant qu'il ne migre à l'extérieur de la prostate et conclut qu'il est d'importance primordiale de mettre en évidence le cancer à ce stade. Malheureusement aucune donnée de la littérature ne permet de dire pour un malade précis le temps écoulé entre l'apparition de la première cellule néoplasique et la possibilité de détection d'un cancer de la prostate. Toute l'argumentation de F. Labrie repose sur le fait que $80 \%$ des cancers découverts dans cette étude l'ont été dans le groupe de 191 hommes sur $1002(19,1 \%)$ ayant un PSA sérique au-dessus de 8 microgrammes par litre. Sur cette base il se fait le champion de la pratique du dosage de PSA chez tous les hommes après 45 ans une fois par an et il calcule que le coût de $m / s n^{\circ} 7$, vol. 8, seplembre 92
1500 US dollars pour mettre en évidence un cas de cancer de la prostate est peu élevé. En fait le coût serait sensiblement plus élevé car, avec la répétition annuelle du dosage de PSA, le nombre de cas dépistés diminuera nécessairement et le calcul ne tient pas compte de la fréquence des cancers impossibles à dépister, qui est par définition inconnue.

Le raisonnement de F. Labrie méconnaît un certain nombre de points qui font l'objet de nombreuses discussions urologiques.

1. L'âge du dépistage : parmi les 57 patients chez qui un cancer de la prostate est mis en évidence, aucun n'a entre 45 et 50 ans, deux ont entre 50 et 55 ans, sept entre 55 et 60 ans, 30 entre 60 et 70 ans, 18 entre 70 et 80 ans. Bien qu'on n'ait pas d'information sur la pyramide des âges de la population, étudiée, il apparaît déjà que seule la tranche entre 60 et 70 ans est suffisamment importante pour justifier peut-être un dépistage systématique.

2. Le nombre de biopsies qui n'aboutissent pas au diagnostic de cancer prostatique. Cooner et al. dans le Journal of Urology (1990 ; 143 : 1146-90) a bien montré que dans la catégorie de population ayant un PSA entre 4 et $10 \mathrm{ng} / \mathrm{ml}$, il fallait six biopsies pour faire le diagnostic d'un seul cancer. Même si les techniques de biopsie ont évolué vers une agressivité de moins en moins importante, les complications à craindre sur une grande population dépistée ne doit pas être négligée.

3. Sans doute l'argument le plus important est qu'on n'est pas vraiment certain du bénéfice apporté à un homme donné en dépistant chez lui un cancer de la prostate limité à la glande. Diagnostiquer des cancers en plus grand nombre et plus précocement n'améliore pas nécessairement la durée, ni la qualité de la survie. La série de Johanson publiée dans le JAMA d'avril 1992 est révélatrice à cet égard. En effet, sur une série de cancers limités de 220 cas suivis pendant une période de 81 à 103 mois, la survie à 10 ans est de $86,8 \%$, tout à fait comparable à celle d'un sous-groupe où était pratiqué une prostatectomie radicale.

Il est donc "prématuré " de suivre Fernand Labrie lorsqu'il écrit, " il est cependant reconnu que le cancer de la prostate découvert au stade précoce et non traité évolue vers les métastases et le décès dans une proportion importante de cas ". Le mot " importante" mériterait d'être défini. Les bases de son calcul selon lequel " le diagnostic et le traitement précoce et efficace du cancer de la prostate pourrait alors potentiellement ajouter en moyenne au moins 10 années de vie à chaque homme ainsi traité ", n'est ni explicité, ni étayé par les statistiques de survie actuellement disponibles.

Ainsi, la très intéressante étude de F. Labrie est-elle une contribution à la connaissance du cancer de la prostate, mais ne résoud pas le problème d'une stratégie de dépistage. Il y a vraisemblablement comme l'indique F. Labrie, urgence à évaluer l'efficacité des traitements précoces à visée curatrice, préalable indispensable à la mise en œuvre d'un dépistage systématique. Cela étant, le tirage au sort d'une série de patients entre l'abstention thérapeutique et la prostatectomie radicale est difficile à proposer au plan de l'éthique

P. Robel : professeur, directeur de recherche à l'Inserm. A. Jardin : prof esseur, chef du service d'urologie. Hôpital de Bicêtre, Université Paris-Sud, 78, avenue du général Leclerc, 94275 Le Kremlin-Bicêtre, France. 